confirmed HIV-positive. Specificity was high (> 99.8\%) for both tests. Four early infections could only be detected by CMIA and confirmed by RT-PCR whereas RA and immunoblot analysis were still negative.

Discussion Approximately half of the LPHA in NRW offered their clients the RA. Clients deciding for RA were slightly different according to age, gender, risk behaviour and HIV status. It could not be shown so far that by offering RAs the LPHA attracted special risk groups which might otherwise not have been tested for HIV. It needs to be considered that some early HIV infections could be detected by CMIA but not by RA.

\section{P2.059 EVALUATION OF A 2ND GENERATION REAL TIME PCR SYSTEM FOR DIAGNOSIS OF CHLAMYDIA TRACHOMATIS: IMPACT ON LABORATORY WORKFLOW}

doi:10.1136/sextrans-2013-051184.0324

D De Maria, M D'Autilia, M A Latino. S.S. of Bacteriology, O.I.R.M. - Sant'Anna Hospital, Turin, Italy

Background The aim of this study was to evaluate the performance and the impact on laboratory workflow of Cepheid Xpert CT, a new generation of Real Time PCR test that provides results in $90 \mathrm{~min}$, with only $2 \mathrm{~min}$ of hands on time in comparison with traditional molecular method used (Alert q-PCR ELItech, Nanogen).

Methods 101 selected women (<25 and; 15\% > 25 years old with risk factors) have been enrolled for this evaluation. Performances and laboratory workflow were compared: Xpert CT was run on GeneXpert System and Alert q-PCR on ABI 7300 (Life Technologies). Residual samples (500 uL UTM endocervical swabs) previously tested with Alert q-PCR have been used for the Xpert CT assay.

Results On a total of 101 samples, 98 were concordant and 3 were discordant: 2 were positive with Xpert CT and negative for Alert q-PCR and 1 was positive with Alert q-PCR and negative with Xpert CT. It was appreciated the value of Sample Adequacy Control (SAC) in Xpert CT, that presented low Ct value (below 20) in case of severe infection. Laboratory Workflow: GeneXpert ${ }^{\circledR}$ steps $n=23$ for extraction, amplification and detection (the whole RT-PCR process happened inside the cartridge), TAT $90 \mathrm{~min}$. Alert q-PCR for a run of 24 samples: extraction steps $n=253$, amplification and detection steps $\mathrm{n}=286$, hands on time $70 \mathrm{~min}$, extraction $55 \mathrm{~min}$, amplification and detection $2 \mathrm{~h}$, TAT $4 \mathrm{~h}$

Conclusions GX simplified the laboratory workflow ensuring standardisation, accuracy and reliability of analytical data. The value of SAC supports the quality of sampling to avoid false negative results due to insufficient cells detected. Need evaluation for discrepancy results.

\section{P2.060 MULTIPLEX REAL-TIME PCR FOR THE SIMULTANEOUS DETECTION OF 7 SEXUALLY TRANSMITTED PATHOGENS REVEALS A HIGH RATE OF MULTIPLE INFECTIONS}

doi:10.1136/sextrans-2013-051184.0325

'D Danioth, ${ }^{2} \mathrm{M}$ Affolter, ${ }^{3} \mathrm{~F}$ Imeri, 'M Altwegg. 'Bioanalytica, Lucerne, Switzerland; ${ }^{2} \mathrm{MCL}$ Medical Laboratories, Berne, Switzerland; 3 Laborgemeinschaft 1, Zurich, Switzerland

Background Sexually transmitted infections are caused by a relatively well defined group of pathogens. Their individual detection using cultural and molecular techniques is time-consuming and costly. Multiplex real-time PCR is a rapid and more cost-effective alternative and allows the detection of multiple infections.

Methods We have validated the Anyplex II ${ }^{\text {TM }}$ STI-7, a semiquantitative, highly multiplexed real-time PCR kit (Seegene), using a selection of specimens positive by routine methods (culture, PCR, cytology) for at least one of the 7 different targets. Specimens were assumed to be negative for those parameters not previously tested.
DNA was isolated using the easyMAG ${ }^{\circledR}$ (bioMérieux) followed by melting curve analysis-based PCR on a CFX96 ${ }^{\mathrm{TM}}$ thermocycler (Bio$\mathrm{Rad}$ ) and automatic data interpretation with the Seegene Viewer software. Discrepant results were resolved with independent molecular tests.

Results Resolved results showed 100\% sensitivity and specificity for Chlamydia trachomatis (17 positive/73 negative specimens), Neisseria gonorrhoeae (13/71), Trichomonas vaginalis (6/84), Mycoplasma genitalium (18/72) and Mycoplasma hominis (30/60). For Ureaplasma species (57/30) 100\% sensitivity and $93.3 \%$ specificity were observed. The STI-7 test (the only test capable of separating the two major species) revealed that among the 57 Ureaplasma-positive specimens 7 (12.3\%) were positive for U. urealyticum, 39 (68.4\%) for U. parvum and $11(19.3 \%)$ for both species. Often, 2 or more targets were detected, e.g. of the 17 C. trachomatis-positive specimens 8 were positive for 1 and 7 for 2 additional organisms. Ureaplasmas were the most prevalent species being present in about $2 / 3$ of the specimens.

Conclusion We conclude that the STI-7 multiplex PCR is a rapid and reliable test for the simultaneous detection of the most important sexually transmitted pathogens providing an efficient means for a more thorough evaluation of the clinical significance of the various organisms.

\section{P2.061 ACCURACY OF SYNDROMIC DIAGNOSIS (SD) FOR VAGINAL DYSCHARGE AND CERVICITIS IN WOMEN OF REPRODUCTIVE AGE IN BOGOTA, COLOMBIA}

doi:10.1136/sextrans-2013-051184.0326

'J Tolosa, ${ }^{2} \mathrm{~A}$ Rodriguez, ${ }^{3} \mathrm{E}$ Angel Muller, ${ }^{4} \mathrm{~A}$ Ruiz Parra, ${ }^{5} \mathrm{H}$ Gaitan Duarte. ${ }^{1}$ Oregon and Health Science University., Portland, OR, United States; ${ }^{2}$ Clinical Research Institute, Universidad Nacional de Colombia, Bogota, Colombia; ${ }^{3}$ Department of Obstetrics and Gynecology, Universidad Nacional de Colombia, Bogota, Colombia; ${ }^{4}$ Clinical Research Institute, Universidad Nacional de Colombia, Bogota, Colombia; ${ }^{5}$ Clinical Research Institute, Clinical Research Institute, Universidad Nacional de Colombia, Bogota, Colombia

Objective Determine the diagnostic accuracy of the symptoms and signs used in the syndromic diagnosis of low genital tract infections (LGTIs) in symptomatic women.

Materials and Methods: A diagnostic validity study of sexually active women (14-49 years old) consulting with symptoms of LGTI at three outpatient facilities in Bogota, Colombia were recruited in 2010. Exclusion criteria: hysterectomy, pregnancy or antibiotics in the 7 previous days. Symptoms and signs and syndromic diagnosis were evaluated by a physician for two syndromes: Vaginal discharge caused by Bacterial vaginosis (BV) Candida albicans (CA) and Trichomonas vaginalis (TV) and Cervicitis caused by $\mathrm{N}$. gonorrhoeae (NG), and $\mathrm{C}$ trachomatis (CT). Those were compared against PCR for CT and NG; Nugent's criteria for BV; wet smear for TV and blood agar culture for CA. Sensitivity, specificity, LR (+), LR (-) for each syndrome and its symptoms and signs were calculated Results 1372 subjects were evaluated. The prevalence of NG and CT was 1.3\% (18/1372) and 9.1\% (125/1372); for BV, TV and CA infection was $39.9 \%(548 / 1372), 0.8 \%(11 / 1372)$ and $11.1 \%$ (152/1372). Sensitivity and Specificity are for syndromic approach for cervicitis 13.3\%(CI 95 8.2-20) and 90.9\% (CI95 89.1-92.4) respectively. Vaginal discharge is the sign with the most sensitivity for cervicitis and BV, TV and CA infections: $93 \%$ and $78 \%$, respectively. In cervicitis, the most specific sign is mucopurulent cervical discharge $(91.8 \%)$ and for BV, TV and CA infections is erythema valvular (68\%)

Conclusions SD for vaginal discharge syndrome has a high sensitivity and a low specificity resulting in a high rate of unnecessary antibiotic treatment (64.9\%). SD for cervicitis, has a low sensitivity and high specificity resulting in a high \% of false negatives and lack of needed antibiotic treatment in $86.7 \%$. SD alone is an ineffective strategy for LGTIs. 